\title{
The Effect of Memory Size on the Evolutionary Stability of Strategies in Iterated Prisoner's Dilemma
}

Article in IEEE Transactions on Evolutionary Computation · October 2013

Impact Factor: $3.65 \cdot$ DOI: 10.1109/TEVC.2013.2286492

CITATION

1

2 authors:

Jiawei Li

University of Stirling

42 PUBLICATIONS 315 CITATIONS

SEE PROFILE
READS

32

Graham Kendall

University of Nottingham, Malaysia Campus

301 PUBLICATIONS $\quad 6,375$ CITATIONS

SEE PROFILE 


\title{
The effect of memory size on the evolutionary stability of strategies in iterated prisoner's dilemma
}

\author{
Jiawei Li, Member, IEEE, and Graham Kendall, Senior Member, IEEE
}

\begin{abstract}
The iterated prisoner's dilemma is an ideal model for the evolution of cooperation among the payoff-maximizing individuals. It has attracted wide interest to develop novel strategies since the success of tit-for-tat in Axelrod's iterated prisoner's dilemma competitions. Every strategy for iterated prisoner's dilemma utilizes a certain length of historical interactions with the opponent, which is regarded as the size of the memory, in making its choices. Intuitively, longer memory strategies must have an advantage over shorter memory strategies. In practice, however, most of the well-known strategies are short memory strategies that utilize only the recent history of previous interactions. In this paper, the effect of the memory size of strategies on their evolutionary stability in both infinite length and indefinite length $n$-person iterated prisoner's dilemma is studied. Based on the concept of a counter strategy, we develop a theoretical methodology to evaluate the evolutionary stability of strategies and prove that longer memory strategies outperform shorter memory strategies statistically in the sense of evolutionary stability. We also give an example of a memory-two strategy to show how the theoretical study of evolutionary stability assists in developing novel strategies.
\end{abstract}

Index Terms-Evolutionary stability, strategies, iterated prisoner's dilemma, game theory.

\section{INTRODUCTION}

$\mathbf{T}$ HE prisoner's dilemma is a non-zero-sum game in which two players try to maximize their payoff by cooperating with, or betraying the other player [34], [35]. The payoff matrix of the game is shown in Figure 1.

$$
\begin{array}{cccc} 
& \multicolumn{2}{c}{\text { Prisoner 2 }} \\
& \text { Cooperate } & \text { Defect } \\
\text { Prisoner 1 Cooperate } & (R, R) & (S, T) \\
& \text { Defect } & (T, S) & (P, P)
\end{array}
$$

Fig. 1. Payoff matrix of the Prisoner's Dilemma.

In the payoff matrix, $R, S, T$, and $P$ denote Reward for mutual cooperation, Sucker's payoff, Temptation to defect, and Punishment for mutual defection respectively, and $T>R>$ $P>S$. The constraint motivates each player to play noncooperatively.

J. Li is with the School of Computer Science, ASAP research group, University of Nottingham, Jubilee Campus, Wollaton Road, Nottingham, UK, NG8 1BB. E-mail: jiawei.li@nottingham.ac.uk

G. Kendall is with the School of Computer Science, ASAP Research Group, University of Nottingham, UK. E-mail: graham.kendall@ nottingham.ac.uk and University of Nottingham Malaysia Campus, Jalan Broga, 43500 Semenyih, Selangor Darul Ehsan, Malaysia. E-mail: graham.kendall@ nottingham.edu.my

Manuscript received December 28, 2012; Accepted October 9, 2013.

Copyright (c) 2012 IEEE.
When both players are rational and they make their choice independently, the theoretical outcome of the game is a Nash equilibrium, in which both players choose to defect and each receives a 'Punishment for mutual defection'. It is worse for each player than the outcome they would have received if they had cooperated.

In the Iterated Prisoner's Dilemma (IPD) game, two players have to choose their mutual strategy repeatedly, and they also have memory of their previous behaviors and the behaviors of the opponents. $R>\frac{1}{2}(S+T)$ is set to prevent any incentive to alternate between cooperation and defection. IPD is considered to be an ideal test bed for the evolution of cooperation among selfish individuals and it has attracted wide interest since Robert Axelrod's IPD tournaments and 'The Evolution of Cooperation' [4], [5], [11], [21], [27], [28].

The winner of Axelrod's tournaments was the 'tit-for-tat' (TFT) strategy. TFT starts with cooperation, and then copies the opponent's previous move. Axelrod attributes the success of TFT to its properties of 'nice', 'forgiving', 'retaliating' and 'simple'. However, later research has shown some weaknesses of TFT such as vulnerability to noise and being unable to exploit unconditional cooperators [28], [39]. Since then, researchers have attempted to develop novel strategies that can outperform TFT in either round-robin IPD competitions or evolutionary dynamics and some IPD strategies have been developed, for example, 'win-stay lose shift' [29], 'generousTFT' [33], 'gradual' [6], and very recently 'group strategies' [22], [36], [24], and 'zero-determinant' [31], [37]. Also, evolution of strategies in replicator dynamics with infinite or finite population, spatial, and noisy environments has been studied in [1], [2], [8], [9], [15], [16], [17], [19], [20], [32], [26].

Different IPD strategies may use different lengths of historic interactions. TFT, for example, is a memory-one strategy, only making use of information from the previous stage of interaction in making its next decision. 'Tit-for-two-tats' is a memory-two strategy. Most well known IPD strategies are memory-one and memory-two strategies and only a limited number of strategies use a memory size greater than three.

Intuitively, the players with longer memories can perform at least as well as those with shorter memories. However, it is still not clear whether longer memory strategies outperform shorter memory strategies. Few IPD strategies developed in either experiments or tournaments uses a very long memory. A non-trivial question is whether or not a longer memory grants a strategy advantage in IPD?

Experiments have revealed that memory helps learning and cooperation in evolution [41], [14], [13]. Posch [30] studied win-stay, lose-shift with diverse memory size and showed that win-stay, lose-shift with longer memory performed better than 
those with shorter memory in computer simulations. Ashlock and Roger [3] showed that several strategies utilizing long term memory outperformed control strategies in evolutionary IPD. Press and Dyson [31] proves that the shortest memory strategy sets the rule of 2-player IPD games. That is, longer memory strategies do not have an advantage over shorter memory strategies in 2-player IPD. However, this result cannot be extended to $n$-player $(n>2)$ IPD cases and the memoryone zero-determinant strategies are not evolutionarily stronger than those known IPD strategies [37], [42].

In this study, we investigate the effect of memory size on the evolutionary stability of IPD strategies. In order to evaluate the evolutionary stability of IPD strategies, we develop the concept of a counter strategy. A strategy is a counter strategy against another strategy if it receives no less payoff than any strategy in interacting with the opponent. Based on this concept, a number of theorems which show the relationship between the length of the history a strategy uses and its evolutionary stability are proven. The contributions of this paper include:

$a$. We propose a theoretical methodology to evaluate the evolutionary stability of IPD strategies. Based on the concept of a counter strategy, the evolutionary stability of a strategy is evaluated by whether the strategy is a counter strategy against itself and the probability that the strategy is a counter strategy against an arbitrary strategy.

$b$. The effect of the memory size of IPD strategies on their evolutionary stability is analyzed. We prove that longer memory strategies have an advantage over shorter memory strategies in both infinite length and indefinite length $n$-IPD. A longer memory strategy has a higher probability of winning against an arbitrary strategy than a shorter memory strategy.

The rest of paper is structured as follows. Section 2 introduces the concept of counter strategy and how the evolutionary stability of IPD strategies can be evaluated by means of a counter strategy. Section 3 presents three theorems and their proofs. The theorems show the effect of memory size of strategies on their evolutionary stability. Finally, section 4 has concluding remarks.

\section{Evolutionary Stability OF IPD stRategies}

An indefinite length IPD has a discount rate $\omega(1 \geq \omega>0)$. The game continues with probability $\omega$ and the expected number of iterations of the game are $1 /(1-\omega)$. If $\omega=1$, the game is infinite. In this paper, we focus on $n$-player infinite length and indefinite length IPDs and ignore the finite length IPD. Let $S_{i}(i=1, \ldots n)$ denote the strategy of the $i^{t h}$ player and $E\left(S_{i}, S_{j}\right)$ the payoff of $S_{i}$ playing against strategy $S_{j}$. Then the payoff of $S_{i}$ playing against all $n$ strategies (including playing against itself) can be expressed as $E\left(S_{i}\right)=\sum_{j=1}^{n} E\left(S_{i}, S_{j}\right)$.

\section{A. Counter strategy}

Let $H_{L}$ denote a history of $L$ moves of interactions between two players before the current move. A strategy that makes use of $H_{L}$ is called a memory- $L$ strategy. A player should have at least $L$ length of memory in order to adopt a memory$L$ strategy.
The strategies in IPD can be categorized according to the length of memory they use. Some well known IPD strategies are shown in Table I. Descriptions of all strategies used in this paper can be found in [23]. For those strategies that have variable memory length, the memory length is the longest memory that they use. Let $f\left(H_{L}\right)$ denote a memory-L strategy. The strategy space of a length-L IPD is determined by $\left\{f, H_{L}\right\}$ and the strategy space of an infinite or indefinite length IPD is determined by $\left\{f, H_{\infty}\right\}$.

TABLE I

SOME WELL KNOWN IPD STRATEGIES WITH DIFFERENT SIZE OF MEMORIES.

\begin{tabular}{|c|c|}
\hline Memory-zero & Memory-one \\
\hline \hline Always-cooperate & Tit-for-tat, GRIM triger \\
Always-defect & Renerous TFT, Contrite TFT \\
Rindom & Zero-los-determinant \\
& Memory- $L(L>2)$ \\
\hline \hline Memory-two & Fortress \\
\hline \hline Tit-for-two-tats & Prober \\
Two-tits-for-tat & Group strategies \\
& \\
\hline
\end{tabular}

In order to evaluate the evolutionary stability of IPD strategies, we introduce the concept of a counter strategy. A strategy $S$ is a counter strategy (CS) against another strategy $S_{1}$ if, for any strategy $S^{\prime}$

$$
E\left(S, S_{1}\right) \geq E\left(S^{\prime}, S_{1}\right)
$$

A CS receives the highest payoff in playing 2-IPD against another strategy. In an indefinite length 2-IPD, for example, Always-defect (AllD) is a CS against always-cooperate; TFT is a CS against TFT. For an arbitrary strategy, there must be at least one CS against it.

Specially, strategy $S$ is a CS against itself (CSI) if, for any strategy $S^{\prime}$

$$
E(S, S) \geq E\left(S^{\prime}, S\right)
$$

A strategy $S$ is a CS against a population of strategies $\left\{S_{i}\right\}$ $(i=1, \ldots, n)$ if, for any $S^{\prime}$ and $S_{i}$, there is

$$
E\left(S, S_{i}\right) \geq E\left(S^{\prime}, S_{i}\right)
$$

If a strategy is a CS against a population, it is a CS against any member of the population and thus it receives the highest payoff in playing $n$-IPD against the population.

There is an equivalence between a CS against a mixed strategy and a CS against a population. Assume that $S$ is a CS against a population of $n$ strategies $\left\{S_{i}\right\}$. Consider the mixed strategy $\bar{S}$ that assigns probabilities $p_{i}=1 / n$ to $S_{i}$,

$$
\bar{S}=\left\{\begin{array}{cc}
S_{1}, & p_{1} \\
S_{2}, & p_{2} \\
\cdots & \\
S_{n}, & p_{n}
\end{array}\right.
$$

The expected payoff for $S$ playing against $\bar{S}$ is,

$$
\begin{aligned}
E(S, \bar{S}) & =p_{1} E\left(S, S_{1}\right)+p_{2} E\left(S, S_{2}\right)+\cdots+p_{n} E\left(S, S_{n}\right) \\
& =\frac{1}{n} \sum_{i=1}^{n} E\left(S, S_{i}\right)
\end{aligned}
$$


According to the definition of a CS, we have for any $S^{\prime}$,

$$
E(S, \bar{S}) \geq \frac{1}{n} \sum_{i=1}^{n} E\left(S^{\prime}, S_{i}\right)=E\left(S^{\prime}, \bar{S}\right)
$$

Thus, $S$ is also a CS against $\bar{S}$. On the other hand, if a strategy is a CS against a mixed strategy, it must be a CS against a population that contains all pure strategies of the mixed strategy. As shown in the following subsection, the concepts of CSs can be used to evaluate the evolutionary stability of IPD strategies.

\section{B. Evolutionary stability}

An evolutionarily stable stategy (ESS) is a strategy such that, if all the members of a population adopt it, then no mutant strategy can invade the population under the influence of natural selection. According to [25] the condition for a strategy $S$ to be ESS is that for any $S^{\prime}$,

$$
E(S, S) \geq E\left(S^{\prime}, S\right)
$$

This condition is actually equivalent to a Nash equilibrium in a two-player IPD. In order for a homogeneous population to resist invasion of mutant strategies, a more restrictive condition is defined. According to [38], the condition for a strategy $S$ to be ESS is that for any $S^{\prime}$,

$$
\begin{aligned}
& E(S, S) \geq E\left(S^{\prime}, S\right) \\
& E\left(S, S^{\prime}\right)>E\left(S^{\prime}, S^{\prime}\right)
\end{aligned}
$$

This condition guarantees that an ESS always outperforms mutant strategies so that a homogeneous population can be maintained in evolutionary dynamics. However, most evolutionary algorithms for IPD do not satisfy the hypothesis of ESS and it has been proven that no strategy is ESS in infinite length or indefinite length $n$-player IPD [7], [40]. Except in specific situations, the condition of ESS cannot be used to analyze the evolutionary stability of IPD strategies.

There is a relationship between CS and ESS. A strategy is ESS if it is the only CS against all IPD strategies. Assume that $S$ is a CS against all strategies. For any strategy $S^{\prime}$, we have

$$
E(S, S) \geq E\left(S^{\prime}, S\right)
$$

and

$$
E\left(S, S^{\prime}\right) \geq E\left(S^{\prime}, S^{\prime}\right)
$$

Comparing the inequalities with (5), we know that $S$ is ESS if it is the unique CS against all strategies. Since the condition of ESS is too strict, we need another criterion to measure the evolutionary stability of IPD strategies.

It is easy to verify that any CSI is Maynard Smith's definition of ESS. If strategy $S$ is a CSI, (4) always holds for any strategy $S^{\prime}$. There is an infinite set of CSIs in $n$-IPD. Some well known strategies, for example, AllD, TFT, and twotits-for-tat (TTFT) are such strategies. A CSI is an equilibrium choice for a player and thus it is superior to any non-CSI in maintaining a homogeneous population.

In order to evaluate the evolutionary stability of CSIs, we need another criterion. Given a strategy $S$, let $p(S)$ denote the probability that $S$ is a CS against an arbitrary IPD strategy. A strategy with a high value of $p(S)$ is more likely to win in an $n$-IPD competition or evolution. We call $p(S)$ the evolutionary stability value of $S$. For any strategy, there is $1 \geq p(S) \geq 0$. A strategy is ESS if it is the only strategy satisfying $p(S)=1$.

Here we give an example of computing the evolutionary stability value of a random strategy. Let $S$ denote a random strategy that chooses between $\mathrm{C}$ and $\mathrm{D}$ in every move, and $S^{\prime}$ an arbitrary strategy. In an indefinite length $n$-IPD with discount rate $\omega$, the expected length of interaction between $S$ and $S^{\prime}$ is $1 /(1-\omega)$. It needs to make the 'correct' choice in every move in order for $S$ to be a CS against $S^{\prime}$. Because $S$ is a random strategy, the probability that it makes the 'correct' choice in each move is 0.5 . Therefore, the probability that $S$ is a CS against $S^{\prime}$ is $0.5^{\frac{1}{1-\omega}}$.

Different IPD strategies can be compared according to whether or not they are CSI and their evolutionary stability values. A CSI outperforms any non-CSI and a CSI with a higher evolutionary stability value outperforms another CSI with a lower evolutionary stability value. In the following section, several theorems are given to show that longer memory strategies outperform shorter memory strategies.

\section{THE EFFECT OF MEMORY LENGTH ON EVOLUTIONARY STABILITY}

Different IPD strategies use different history lengths of interactions among players to determine their choices. In a finite length IPD that iterates exactly $L$ rounds, the history length a strategy can access is at most $L$. In an infinite length IPD, however, the history length is infinite. A non-trivial question is whether the full history length is useful for an individual player to interact with others optimally?

It has been proven that every finite history length is possible to occur in an infinite length $n$-IPD. This is expressed as the following theorem [40].

Theorem 1: In the infinite length $n$-IPD where the probability of further interaction is sufficiently high, every finite history of interactions among the $n$ players occurs with positive probability in any evolutionarily stable mixture of pure strategies.

It is easy to verify that Theorem 1 also holds in an indefinite length $n$-IPD. In an indefinite length IPD, the probability that the game continues to any limited number of stages is positive. Thus, every finite history occurs with positive probability.

A direct conclusion from Theorem 1 is that every finite history may be useful in order for a strategy to interact with an arbitrary strategy optimally. As to the relationship between the history length a strategy uses and its evolutionary stability, we have the following theorems.

Theorem 2: For any strategy that uses a limited history length, there always exist some strategies with longer memory against which the strategy cannot be a counter strategy.

Proof: Consider a memory- $L$ strategy $S$. Let $H_{L}$ denote a specific $L$ length history of interactions between $S$ and an 
arbitrary strategy $S_{1}$.

a. If $S$ is a pure strategy, without loss of generality, assume that $S$ responds D (or C) to $H_{L}$. Let $H_{L+1}^{\prime}$ denote a $L+1$ length history in which the first $L$ moves are the same as $H_{L}$ and the opponent plays $\mathrm{D}$ (or C) at the last move. Another strategy $S_{2}$ is defined as below,

$$
S_{2}=\left\{\begin{aligned}
S_{1} & \text { in the first } \mathrm{L} \text { moves } \\
\text { always }- \text { defect } & \text { if } H_{L+1}^{\prime} \\
\text { cooperate } & \text { otherwise }
\end{aligned}\right.
$$

$S_{2}$ is a memory- $(L+1)$ strategy. We now prove that $S$ is not a CS against $S_{2}$. Consider a strategy $S^{\prime}$ defined as below,

$$
S^{\prime}= \begin{cases}S & \text { in the first } \mathrm{L} \text { moves } \\ C & \text { if } H_{L} \\ S & \text { otherwise }\end{cases}
$$

In the 2-IPD between $S^{\prime}$ and $S_{2}, S_{2}$ plays cooperate after $L+1$ moves, while in the 2-IPD between $S$ and $S_{2}, S_{2}$ plays always-defect after $L+1$ moves. It is easy to verify that $E\left(S, S_{2}\right)<E\left(S^{\prime}, S_{2}\right)$.

b. If $S$ is a mixed strategy, without loss of generality, assume that $S$ responds D to $H_{L}$ with probability $p>0$ (responds $\mathrm{C}$ with probability $1-p$ ). Let $H_{L+1}^{\prime}$ denote a $L+1$ length history in which the first $L$ moves are the same as $H_{L}$ and the opponent plays D at the last move. Consider two strategies $S_{2}$ and $S^{\prime}$ as defined in (6) and (7). There is $E\left(S, S_{2}\right)<E\left(S^{\prime}, S_{2}\right)$.

Thus, $S$ is not a CS against $S_{2}$.

Theorem 3: Any strategy that uses a limited history length cannot be an ESS in an infinite length or indefinite length $n$-IPD.

Proof: It is a direct conclusion from theorem 2 that any limited memory strategy cannot be ESS in an infinite length $n$-IPD. Consider a memory- $L$ strategy $S$ in an indefinite length $n$-IPD with discount rate $\omega>0$. Since the probability that the game continues more than $L$ stages is positive, there is always a positive probability that $S$ meets a memory- $(L+1)$ strategy against which $S$ is not a CS. Thus, $S$ cannot be an ESS.

Lemma 1: If a strategy is a CS against a memory- $L$ strategy, this strategy will eventually play a periodic sequence in the 2IPD against the memory- $L$ strategy and the period will be $\leq L$.

Proof: Consider an arbitrary memory- $L$ strategy $S_{1} . S_{2}$ is a CS against $S_{1}$. If $L=0, S_{2}$ must be always-defect and Lemma 1 holds. We only need to consider the case of $L>0$.

a. Assume that $S_{1}$ is a pure strategy. Let $h_{1}$ and $h_{2}$ denote two continuous length- $L$ history of interactions between $S_{1}$ and $S_{2}$, $s_{1}$ and $s_{2}$ denote two sequences of moves played by $S_{1}$ in $h_{1}$ and $h_{2}$ respectively, and $l_{1}$ and $l_{2}$ denote two sequences of moves played by $S_{2}$ in $h_{1}$ and $h_{2}$ respectively, as shown in Fig.2.

Because $S_{1}$ is a memory- $L$ pure strategy, the first move of $s_{2}$ is determined by $s_{1}$ and $l_{1}$. Since $S_{2}$ is CS against $S_{1}, l_{1}$ must be the optimal sequence of moves such that $S_{2}$ receives the highest payoff in playing against both $s_{1}$ and the first

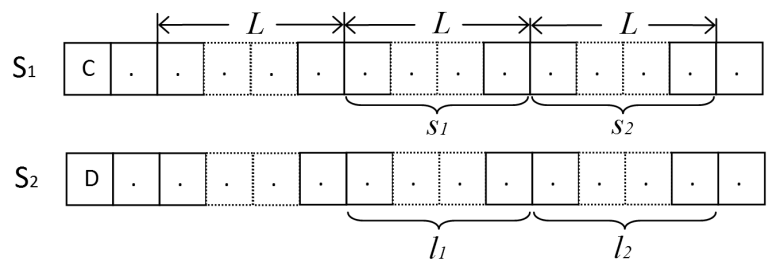

Fig. 2. Two continuous length- $L$ sequences of moves in the interaction between $S_{1}$ and $S_{2}$.

move of $s_{2}$. Given $s_{1}, l_{1}$ and the first move of $s_{2}$, the second move of $s_{2}$ is determined, ... and etc. Given $s_{1}$ and $l_{1}$, each move of $s_{2}$ is determined and thus $l_{2}$ is determined. Since $l_{1}$ is the payoff-maximizing sequence, there must be $l_{2}=l_{1}$.

b. If $S_{1}$ is a mixed strategy, without loss of generality, assume that $S_{1}$ assigns probabilities over $m$ pure strategies $S_{i}^{\prime}$, each of which is memory- $L$ or less.

$$
S_{1}=\left\{\begin{array}{cc}
S_{1}^{\prime}, & q_{1} \\
S_{2}^{\prime}, & q_{2} \\
\cdots & \\
S_{m}^{\prime}, & q_{m}
\end{array}\right.
$$

$S_{2}$ is a CS against any pure strategy $S_{i}^{\prime}(i=1, \ldots m)$. According to the proof in section a, $S_{2}$ will eventually play a periodic sequence against any $S_{i}^{\prime}$ and the period will be $\leq L$. $\square$

Theorem 4: There are always longer memory strategies that have a higher probability of being a CS against an arbitrary strategy than a shorter memory strategy in an infinite length or indefinite length $n$-IPD.

Proof: We first consider the case of infinite length $n$-IPD. Let $S^{\prime}$ be an arbitrary strategy. If $S^{\prime}$ is a memory- $L$ strategy, a CS against $S^{\prime}$ must play a periodic sequence with period $\leq L$ according to Lemma 1. Without loss of generality, let $q_{i}(i=$ $1, \ldots L)$ denote the probability that the CS plays a sequence whose period is equivalent to $i$. There is $q_{i} \geq 0$ and $\sum_{i=1}^{L} q_{i}=1$.

Let $p_{L}(S)$ denote the probability of $S$ being a CS against an arbitrary memory- $L$ strategy. For a memory-zero strategy $S_{0}$, there is $p_{L}\left(S_{0}\right)=\frac{1}{2} q_{1}$ if $S_{0}$ plays a sequence of pure C (or D). If $S_{0}$ plays a periodic sequence whose period is equivalent to 2 , we have $p_{L}\left(S_{0}\right)=\frac{1}{4} q_{2}$, and so on.

$$
p_{L}\left(S_{0}\right)=\left\{\begin{aligned}
\frac{1}{2} q_{1}, & \text { if } S_{0} \text { plays a period-1 sequence } \\
\frac{1}{4} q_{2}, & \text { if } S_{0} \text { plays a period-2 sequence } \\
\ldots & \\
\frac{1}{2^{L}} q_{L}, & \text { if } S_{0} \text { plays a period-L sequence }
\end{aligned}\right.
$$

Thus, the highest value of $p_{L}()$ for a memory-zero strategy is,

$$
p_{L}\left(S_{0}\right)=\max \left(\frac{1}{2} q_{1}, \frac{1}{4} q_{2}, \ldots, \frac{1}{2^{L}} q_{L}\right)
$$

A memory-one strategy can shift between a determined sequence and a period-2 sequence. The highest value of $p_{L}()$ for a memory-one strategy is,

$$
p_{L}\left(S_{1}\right)=\max \left(\frac{1}{2} q_{1}+\frac{1}{4} q_{2}, \frac{1}{8} q_{3}+\frac{1}{4} q_{2}, \ldots, \frac{1}{2^{L}} q_{L}+\frac{1}{4} q_{2}\right)
$$


Similarly, there are $p_{L}\left(S_{L}\right)=\frac{1}{2} q_{1}+\frac{1}{4} q_{2}+\ldots+\frac{1}{2^{L}} q_{L}$, where $S_{L}$ is the memory- $L$ strategy that has the highest value of $p_{L}()$.

For any memory- $K(K \geq L)$ strategy, the highest value of $p_{L}\left(S_{K}\right)$ is,

$$
p_{L}\left(S_{K}\right)=\frac{1}{2} q_{1}+\frac{1}{4} q_{2}+\ldots+\frac{1}{2^{L}} q_{L}
$$

Thus, we have,

$$
\left\{\begin{array}{l}
p_{L}\left(S_{L}\right)>p_{L}\left(S_{L-1}\right)>\ldots>p_{L}\left(S_{1}\right)>p_{L}\left(S_{0}\right) \\
\left.p_{L}\left(S_{K}\right)=p_{L}\left(S_{L}\right) \quad \text { (for any } K \geq L\right)
\end{array}\right.
$$

Because $S^{\prime}$ is an arbitrary strategy, without loss of generality, assume that the probability that $S^{\prime}$ is a memory- $i$ strategy is $q_{i}^{\prime}$. There is $q_{i}^{\prime} \geq 0$ and $\sum_{i=0}^{\infty} q_{i}^{\prime}=1$.

$$
p(S)=\sum_{i=0}^{\infty} q_{i}^{\prime} p_{i}(S)
$$

Let $S_{L}$ be the memory- $L$ strategy that has the highest value of $p()$ in the set of all memory-L strategies, $S_{L+1}$ be the memory- $(L+l)$ strategy that has the highest value of $p()$ in the set of all memory- $(L+l)$ strategies. There is,

$$
\begin{aligned}
p\left(S_{L}\right) & =\sum_{i=0}^{\infty} q_{i}^{\prime} p_{i}\left(S_{L}\right) \\
p\left(S_{L+1}\right) & =\sum_{i=0}^{\infty} q_{i}^{\prime} p_{i}\left(S_{L+1}\right)
\end{aligned}
$$

According to (8), we have,

$$
\begin{cases}p_{i}\left(S_{L}\right)<p_{i}\left(S_{L+1}\right) & \text { (if } i>L) \\ p_{i}\left(S_{L}\right)=p_{i}\left(S_{L+1}\right) & \text { (if } i \leq L)\end{cases}
$$

Thus, there must be $p\left(S_{L+1}\right)>p\left(S_{L}\right)$ for any limited number $L$ in an infinite length $n$-IPD.

In an indefinite length $n$-IPD, the probability that the game continues more than any limited number of stages is positive. There is always a positive probability that the game continues $i$ stages such that $p_{i}\left(S_{L}\right)<p_{i}\left(S_{L+1}\right)$ and thus $p\left(S_{L+1}\right)>p\left(S_{L}\right)$ holds in indefinite length $n$-IPD.

\section{EXAMPLE OF A MEMORY-2 STRATEgY}

In this section, we use a memory-2 (MEM2) strategy to show how the theoretical analysis in the previous section assists in developing IPD strategies. MEM2 behaves like TFT in the first two moves and then it shifts among three strategies, AllD, TFT and TFTT, according to the interactions with the opponent in the last two moves. The logic for MEM2 to choose which strategy to play based on the following rules.

A. If the payoff in two moves is $2 R$ (two mutual cooperations), then play TFT in the following two moves.

B. If the payoff is $T+S$, then play TFTT in the following two moves.

C. In all other cases, play AllD in the following two moves.

D. If AllD has been chosen twice, always play AllD.

MEM2 will cooperate with cooperative strategies according to Rule A, and it can restore cooperation from an occasional defection because of Rule B. Rule D makes sure that MEM2 defects against those periodic or random strategies. It is easy to verify that MEM2 is a CSI strategy.

We run a round-robin IPD competition with 19 strategies, which include MEM2, AllD, TFT, TFTT, and some strategies that have appeared in research papers. The strategies play an IPD with each other and the discount rate of IPD is 0.98 , which means that the average length of the IPD is 50 moves. The scores (average payoff per move) are listed in Table 2. MEM2 receives the highest payoff, significantly higher than other CSI strategies.

TABLE II

RESULT OF A ROUND-ROBIN IPD COMPETITION WITH 19 STRATEGIES.

\begin{tabular}{|c|c||c|c|}
\hline Strategy & Score & Strategy & Score \\
\hline \hline MEM2 & 3.045 & PAVLOV & 2.362 \\
GRIM & 2.799 & HM & 2.314 \\
GRADUAL & 2.749 & AllC & 2.283 \\
TFT & 2.668 & STFT & 2.246 \\
FBF & 2.608 & PCD & 2.243 \\
GTFT & 2.598 & AllD & 2.221 \\
CTFT & 2.587 & RAND & 2.149 \\
TFTT & 2.529 & RTFT & 2.132 \\
RP & 2.399 & NEG & 2.081 \\
NP & 2.364 & & \\
\hline
\end{tabular}

We also run a series of evolutionary IPD simulations. The initial population contains $x=5,6 \ldots 10$ strategies randomly chosen from the 19 strategies in Table II. Each strategy has 20 identical copies. Stochastic universal sampling is used to select parents for the next generation. The parents simply copy their strategies to produce offspring and no mutation is carried out. An evolutionary IPD is run for 100 generations. As the outcome of any single evolutionary IPD is affected by chance, we repeat each evolutionary IPD with the same $x$ value for 50,000 times, and gather statistics on the outcomes. Two measures, average fitness and average frequency in the population, are used to measure the performance of the strategies. They are average values over the results of 50,000 evolutionary IPDs.

The results of simulations are shown in Figs. 3-6. The average fitness and frequencies of all 19 strategies in the population after 100 generations are shown in Fig. 3 and Fig. 4. MEM2 outperforms other strategies in all settings of $x$. The average fitness and frequency of four strategies, MEM2, AllD, TFT, and TFTT as functions of generation are given in Fig. 5 and Fig. 6. It shows that the fitness of MEM2 is significantly higher than other strategies at the beginning of simulations, which leads to its higher frequency in the population. In most of the simulations, defective strategies became extinct after 50 generations and only cooperative strategies remained in the population. This was the reason why the fitness of some cooperative strategies tended to be equal at the end of evolution.

MEM2 shows an example of integrating several different strategies to form a new CSI strategy. Each strategy has its advantages and disadvantages. AllD is a CS against all memory-zero strategies and it receives low payoffs in interacting with most of the memory-nonzero strategies. TFT is a 


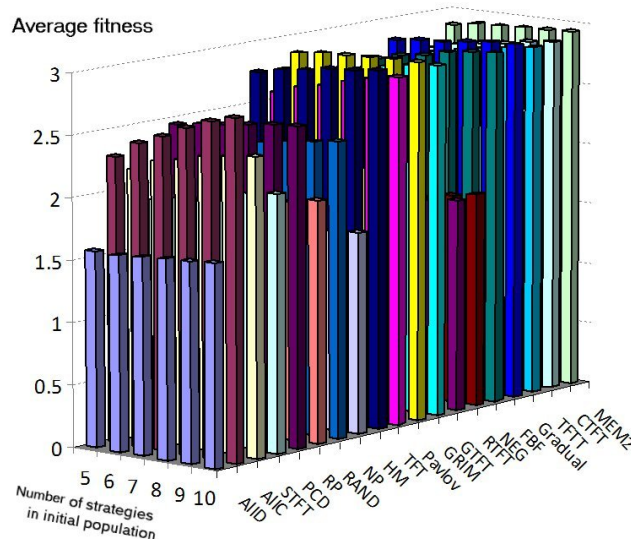

Fig. 3. Average fitness of strategies at generation 100 .

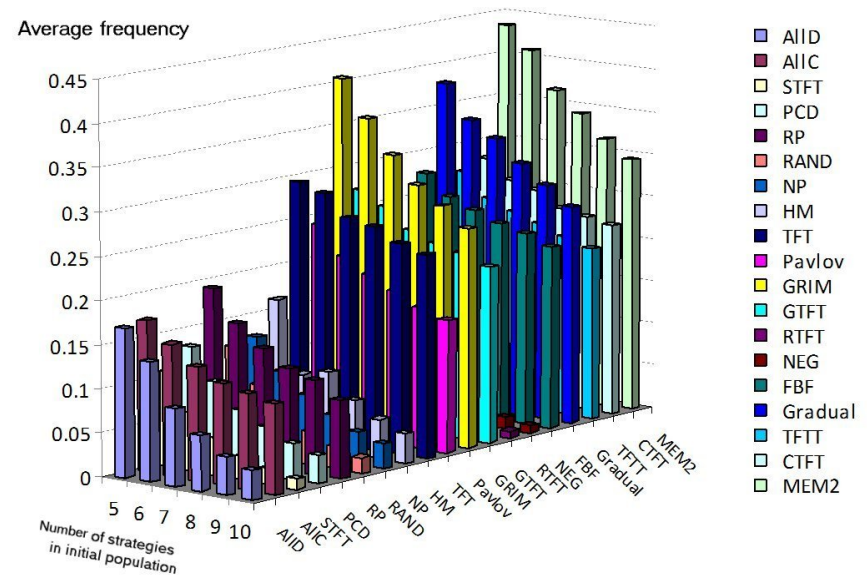

Fig. 4. Average frequencies of strategies at generation 100 .

CS against those strategies that cooperates with the opponent conditionally. A situation that TFT cannot handle well is a long series of mutual retaliations evoked by a single defection. TFTT performs well in this situation by playing one more cooperation. However, it can be exploited by the strategies that alternatively play C and D. MEM2 inherits the advantages of the three strategies and thus outperforms them in evolutionary IPD. The idea of combining different strategies can be used to develop longer memory CSI strategies.

\section{Concluding Remarks}

The condition of ESS is so strict that no strategy can be ESS in infinite length and indefinite length $n$-IPD. With the absence of an ESS, a criterion is needed to evaluate the evolutionary stability of IPD strategies. Based on the concept of a counter strategy, we have proposed a theoretical methodology in which the evolutionary stability of a strategy is evaluated by whether it is CSI and the probability it is a CS against an arbitrary strategy. Different strategies can be compared with each other. The effect of memory size on evolutionary stability is studied by means of this methodology.

The memory length used by a strategy has a significant influence on its evolutionary stability in $n$-IPD. We have proved

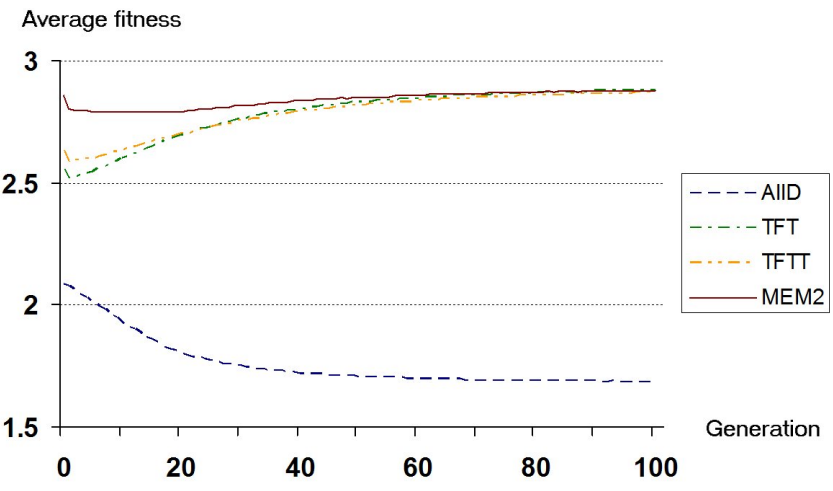

Fig. 5. Fitness of strategies MEM2, AllD, TFT, TFTT $(x=10)$.

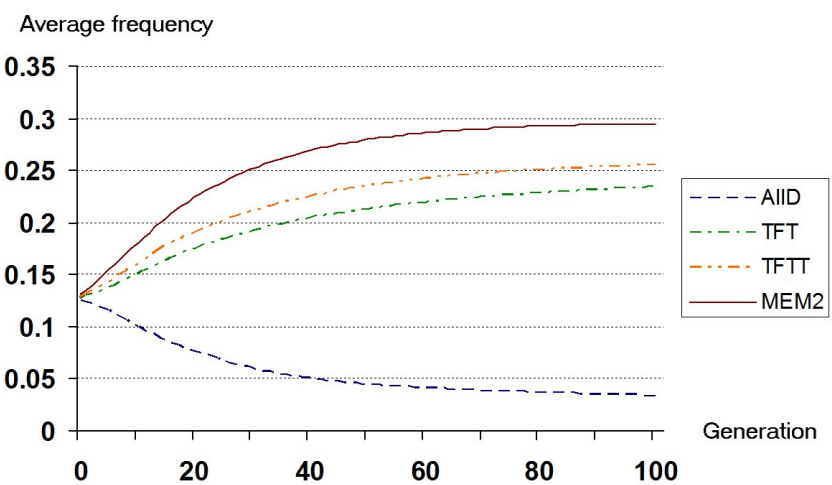

Fig. 6. Frequency of strategies MEM2, AllD, TFT, TFTT $(x=10)$.

that longer memory strategies outperform shorter memory strategies in the sense of evolutionary stability. A well-designed strategy that uses a longer memory statistically receives higher payoffs than shorter memory strategies in interacting with an arbitrary opponent and thus it is more likely to be dominant in evolution.

It may be difficult to theoretically check whether a strategy is a CS against another strategy, especially when both strategies use a long memory, which makes it difficult to compute the evolutionary stability value of an arbitrary strategy. In practice, it is possible to compute approximate evolutionary stability values of IPD strategies by means of statistical methodologies. For example, the performance of strategies in evolution can be measured by running a series of 'races' [18]. The idea is further developed to generalization, a measure for learning performance in co-evolution by using a set of randomly chosen test strategies. It has been proven that an estimated value will approach to the true value as the size of the set of unbiased test strategies increases [10], [12]. As the application of generalization, a statistical methodology that takes into account outcomes across varying $n$-IPD competitions has been used to evaluate the performance of IPD strategies [23]. With this methodology, a series of $n$-IPD competitions are run and the strategies in each competition are randomly chosen from a set of representative strategies. The performance of a strategy is evaluated according to its win rate which is the frequency of achieving the highest payoff in 
a single competition. The win rate of a strategy is considered as an approximation of its evolutionary stability value. In this way, the evolutionary stability value can be computed with greatly reduced computational complexity.

Theorems 2-4 do not necessarily hold in an IPD with noise where the players are assumed to make mistakes occasionally. The effect of noise on the performance of IPD strategies varies although noise generally has a negative effect on the persistence of cooperation. Some strategies are more robust than others in a noisy environment. The effect of memory size in IPDs with noise will be one topic of our future research.

\section{ACKNOWLEDGMENT}

This work was supported by the Engineering and Physical Sciences Research Council UK (EPSRC) grant reference EP/H000968/1.

\section{REFERENCES}

[1] W. Ashlock and D. Ashlock, Changes in prisoner's dilemma strategies over evolutionary time with different population sizes, In Proceedings of IEEE Congress on Evolutionary Computation (CEC 2006), pp. 297-304, 2006.

[2] D. Ashlock and E-Y. Kim, Fingerprinting: Visualization and Automatic Analysis of Prisoner's Dilemma Strategies, IEEE Transactions on Evolutionary Computation, 12(5): 647-659, 2008.

[3] D. Ashlock and N. Roger, The Impact of Long-term Memory in the Iterated Prisoner's Dilemma, in H. Cihan and et al. (eds.) Intelligent Engineering Systems through Artificial Neural Networks, chapter 31, ISBN 9780791802953, 2009.

[4] R. Axelrod, More effective choice in the prisoner's dilemma, Journal of Conflict Resolution, 24(3): 379-403, 1980.

[5] R. Axelrod, The Evolution of Cooperation, New York: Basic Books, 1984.

[6] B. Beaufils, J. Delahaye and P. Mathieu, Our meeting with gradual: a good strategy for the iterated prisoner's dilemma, Artificial Life V, chapter 26, 202-212, 2009.

[7] R. Boyd and J. Lorberbaum, No pure strategy is evolutionarily stable in the repeated Prisoner's Dilemma game, Nature, 327: 58-59, 1987.

[8] R. Chiong and M. Kirley, Effects of Iterated Interactions in Multiplayer Spatial Evolutionary Games, IEEE Transactions on Evolutionary Computation, 16(4): 537-555, 2012.

[9] S. Chong and X. Yao, Behavioral Diversity, Choices and Noise in the Iterated Prisoner's Dilemma, IEEE Transactions on Evolutionary Computation, 9(6): 540-551, 2005.

[10] S. Chong, P. Tino, X. Yao, Measuring Generalization Performance in Coevolutionary Learning, IEEE Transactions on Evolutionary Computation, 12(4): 479-505, 2008.

[11] S. Chong, J. Humble, G. Kendall, J. Li and X. Yao, Iterated Prisoner's Dilemma and Evolutionary Game Theory. In: G. Kendall, X. Yao and S. Chong, eds., The Iterated Prisoners' Dilemma: 20 Years On World Scientific Press, Singapore. 23-62, 2007.

[12] S. Chong, P. Tino, D. Ku and X. Yao, Improving Generalization Performance in Co-evolutionary Learning, IEEE Transactions on Evolutionary Computation, 16(1): 70-85, 2012.

[13] P. Darwen, Co-evolutionary Learning by Automatic Modularisation with Speciation, Ph.D. thesis, University of New South Wales, 1996.

[14] P. Darwen and X. Yao, On evolving robust strategies for iterated prisoner's dilemma, in Lecture Notes in Artificial Intelligence, Progress in Evolutionary Computation, volume 956, Springer, 1995. Pages 276-292.

[15] D. Fogel, The Evolution of Intelligent Decision-Making in Gaming, Cybernetics and Systems, 22(2): 223-236, 1991.

[16] D. Fogel, Evolving Behaviors in the Iterated Prisoner's Dilemma, Evolutionary Computation, 1(1): 77-97, 1993.

[17] D. Fogel, On the Relationship between the Duration of an Encounter and the Evolution of Cooperation in the Iterated Prisoner's Dilemma, Evolutionary Computation, 3(3): 349-363, 1995.

[18] D. Fogel and A. Fraser, Running races with Fraser's recombination, Proceedings of the Congress on Evolutionary Computation, pp. 1217$1222,2000$.
[19] H. Ishibuchi, K. Hoshino, and Y. Nojima, Evolution of strategies in a spatial IPD game with a number of different representation schemes, In Proceedings of IEEE Congress on Evolutionary Computation (CEC 2012), pp. 1-8, 2012.

[20] H. Ishibuchi and N. Namikawa, Evolution of Iterated Prisoner's Dilemma Game Strategies in Structured Demes Under Random Pairing in Game Playing, IEEE Transactions on Evolutionary Computation, 9(6):, 552-561, 2005.

[21] G. Kendall, X. Yao and S. Chong, The Iterated Prisoners' Dilemma 20 Years On. World Scientific, Singapore, Advances in Natural Computation 4, 2007.

[22] J. Li, How to design a strategy to win an IPD tournament, in G. Kendall, $\mathrm{X}$. Yao and S. Chong (eds). The Iterated Prisoners Dilemma: 20 Years On. World Scientific, chapter 4, 2007.

[23] J. Li, P. Hingston and G. Kendall, Engineering Design of Strategies for Winning Iterated Prisoner's Dilemma Competitions. IEEE Transactions on Computational Intelligence and AI in Games, 3(4): 348-360, 2011.

[24] J. Li and G. Kendall, A strategy with novel evolutionary features for iterated prisoner's dilemma. Evolutionary Computation, 17(2): 257-274, 2009.

[25] J. Maynard Smith, Evolution and the Theory of Games, Cambridge University Press, Cambridge, 1982.

[26] S. Mittal and K. Deb, Optimal Strategies of the Iterated Prisoner's Dilemma Problem for Multiple Conflicting Objectives, IEEE TRANSACTIONS ON EVOLUTIONARY COMPUTATION, 13(3): 554-565, 2009.

[27] M. Nowak, Five rules for the evolution of cooperation, Science, 314:1560-1563, 2006.

[28] M. Nowak, A. Sasaki, C. Taylor and D. Fudenberg, Emergence of cooperation and evolutionary stability in finite populations, Nature, 428 : 646-650, 2004.

[29] M. Nowak and K. Sigmund, A strategy of win-stay, lose-shift that outperforms tit-for-tat in the prisoner's dilemma game, Nature, 364: 56$58,1993$.

[30] M. Posch, Win-stay, Lose-shift Strategies for Repeated Games - Memory Length, Aspiration Levels and Noise, Journal of Theoretical Biology, 198(2): 183-195, 1999.

[31] W. Press and F. Dyson, Iterated prisoner's dilemma contains strategies that dominate any evolutionary opponent, PNAS, 109(26): 10409-10413, 2012

[32] H. Quek, K. Tan, C. Goh, and H. Abbass, Evolution and Incremental Learning in the Iterated Prisoner's Dilemma, IEEE TRANSACTIONS ON EVOLUTIONARY COMPUTATION, 13(2): 303-320, 2009.

[33] D. Rand, H. Ohtsuki and M. Nowak, Direct reciprocity with costly punishment: generous tit-for-tat prevails, Journal of Theoretical Biology, 256(1): 45-57, 2009.

[34] A. Rapoport, Optimal policies for the prisoner's dilemma, Technical Report No.50 Psychometric Laboratory, University of North California, MH-10006, 1966.

[35] A. Rapoport, Two-person Game Theory. Dover Publications, New York, 1999.

[36] A. Rogers , R.K. Dash , S.D. Ramchurn, P. Vytelingum and N.R. Jenning, Error-Correcting Codes for Team Coordination within a Noisy Iterated Prisoner's Dilemma Tournament, in G. Kendall, X. Yao and S. Chong (eds). The Iterated Prisoners Dilemma: 20 Years On. World Scientific, chapter 9, 2007

[37] A. Stewart and J. Plotkin, Extortion and cooperation in the prisoner's dilemma, PNAS, 109(26): 10134-10135, 2012.

[38] B. Thomas, On evolutionarily stable sets, Journal of Mathematical Biology, 22:105-115, 1985.

[39] J. Wu and R. Axelrod, How to Cope with Noise in the Iterated Prisoner's Dilemma, Journal of Conflict Resolution, 39(1): 183-189, 1995.

[40] X. Yao, Evolutionary stability in the $n$-person iterated prisoner's dilemma, Biosystems, 37: 189-197, 1996.

[41] X. Yao and P. Darwen, An experimental study of N-person iterated prisoner's dilemma games, Informatica, 18(4): 435-450, 1994.

[42] C. Hilbe, M.A. Nowak and K. Sigmund, The evolution of extortion in iterated Prisoners Dilemma games, Proceedings of the National Academy of Sciences, 110(17): 6913-6918, 2013. 


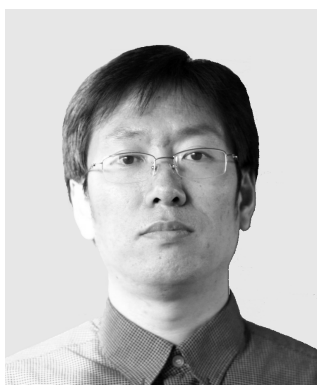

lutionary game theory.

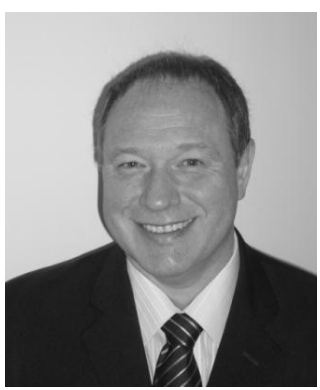

Jiawei Li (M12) received the B.Sc. degree in ship engineering and $\mathrm{Ph}$.D. degree in fluid mechanics from the Harbin Engineering University, Harbin, China in 1992 and 1998 respectively. Dr. Li is currently a research fellow at the School of Computer Science of the University of Nottingham, Nottingham, U.K. His research interests include hyperheuristic, adaptive learning and evo-

Graham Kendall (M03-SM10) received the B.S. degree in computation (first class, honors) from the Institute of Science and Technology, University of Manchester, Manchester, U.K., in 1997 and the Ph.D. degree in computer science from the University of Nottingham, Nottingham, U.K., in 2001. His previous experience includes almost 20 years in the information technology industry where he held both technical and managerial positions. $\mathrm{He}$ is a Professor of Computer Science at the University of Nottingham and is currently based at their Malaysia Campus where he holds the position of Vice-Provost (Research and Knowledge Transfer). He is a Director of two companies (EventMAP Ltd., Nottingham, U.K.; Aptia Solutions Ltd., Nottingham, U.K.) and CEO of two companies (MyRIAD Solutions Sdn Bhd, Malaysia and MyResearch Sdn Bhd, Malaysia).

$\mathrm{He}$ is a Fellow of the Operational Research Society. He is an Associate Editor of nine international journals, including two IEEE journals: the IEEE TRANSACTIONS ON EVOLUTIONARY COMPUTATION and the IEEE TRANSACTIONS ON COMPUTATIONAL INTELLIGENCE AND AI IN GAMES. He chaired the Multidisciplinary International Conference on Scheduling: Theory and Applications in 2003, 2005, 2007, 2009, and 2011, and has chaired several other international conferences, which has included establishing the IEEE Symposium on Computational Intelligence and Games. $\mathrm{He}$ has been awarded externally funded grants worth over $£ 6$ million from a variety of sources including Engineering and Physical Sciences Research Council (EPSRC) and commercial organizations. 